\title{
Research of X-ray Nondestructive Detection System for High-speed running Conveyor Belt with Steel Wire Ropes
}

\author{
Junfeng Wang, Changyun Miao, Yue Cui, Wei Wang \\ School of Information and Communication Engineering, Tianjin Polytechnic University \\ Tianjin 300160, China \\ E-mail: great_seal@163.com \\ Lei Zhou
}

School of Electrical and Computer Engineering, University of Alberta

Alberta t6g92e1, Edmonton, Canada

The research is financed by the National Natural Science Foundation of Tianjin. No.07JCZDJC06300.

\begin{abstract}
The principle of X-ray nondestructive testing (NDT) is analyzed, and the general scheme of the X-ray nondestructive testing system is proposed. The hardware of the system is designed with Xilinx's VIRTEX-4 FPGA in which PowerPC and MAC IP core are embedded, and its peripheral circuits. The network communication software based on TCP/IP protocol, which runs on the hardware platform, is programmed by loading LwIP to PowerPC in XilKernal system. On the basis of analysing image processing algorithm, the image processing software running on the PC is programmed. The NDT of high-speed conveyor belt with steel wire ropes and network transfer function are implemented. It is a strong real-time system with rapid scanning speed, high reliability and remotely nondestructive testing function. The nondestructive detector can be applied to the detection of product line in industry.
\end{abstract}

Keywords: X-ray, NDT, Conveyor belt, PowerPC405, Image processing

\section{Introduction}

Conveyor belt with steel wire ropes, which is one of primary transfer equipments in modern production at home and abroad, is extensively applied in various realms, such as mine mountains, ports and wharfs, etc. As a result of increased load capacity, conveyor belt scratched by barriers and aging, the steel wire rope comes into being tarnishing and cracking steel core or the joint is elongated. Where goes wrong, it can bring into grave worksite/serious accidents, huge economic losses and casualties, and put back safe production badly. (Cheng ,1997). Therefore, detection of conveyor belt with steel wire ropes is a significant research subject at home and abroad.

By the last 1970s, Harrison, Australian scholar, provided a detection method of the conveyor belt with steel wire ropes according to electromagnetic induction principle. In application and practice, our country has developed conveyor belt detection equipment by this principle. However, this method can just detect resultant curve, substantially detect fracture place and degree of steel wire ropes of conveyor belt, can not directly display inner image of steel wire ropes. Therefore this method is lack of accuracy and visualizability. Further more, when the steel wire ropes equips with inner protective screen, this technique cannot be used to detect. By contrast, X-ray nondestructive detector has simple and feasible principle of imaging and great penetrating force, and has widely applied to industry, medical treatment and transportation, and so on. In industry domain, by using high energy X-ray CT, $1 \mathrm{~mm}^{2}$ pore can be found at $100 \mathrm{~mm}^{2}$ iron absorption board, also oil and water in container interior can be differentiated. In medical treatment domain, human body can be examined by X-ray detection technique, and this technique is developed to micromation, high precision and low radiation. In transportation domain, it can be used to inspect luggage in bus stations and aircrafts.

Recently, as modern industry development, nondestructive detector should have high speed, precision, resolution and reliability. Moreover, the present detection systems are mostly at light running, low speed and high cost state, particularly radiant is harmful to human body, so labor protection will be main problem. Against this background above, in this paper network and communication technique is imported to nondestructive detection system. X-ray nondestructive detector for high-speed running conveyor belt with steel wire ropes, which has space resolution of 
$1.5 \mathrm{~mm} \times 1.5 \mathrm{~mm}$ and maximum scanning velocity of $4.3 \mathrm{~m} / \mathrm{s}$, is designed. Through loading LwIP to VIRTEX-4 FPGA that embedded PowerPC and MAC in nondestructive detector, a kind of architecture for the remote access and control networks is constructed based on TCP/IP .This system realizes the purpose of inspecting conveyor belt with steel wire ropes far from radiant source, and is more feasible to radiant protection and human safety.

\section{Principle of $\mathrm{X}$-ray nondestructive detection}

X-ray has a continuous spectrum, and travels in straight lines as visible light. The relationship between its frequency, wavelength and energy is: (Klug, 1974).

$$
\varepsilon=e V=h v=\frac{h c}{\lambda}
$$

where $\varepsilon$ is photon energy, unit is $\mathrm{J} ; e V$ is energy that electron beats to plate target, unit is $\mathrm{J}$; $h$ is Planck's constant, $h=6.63 \times 10^{-34} \mathrm{~J} \cdot \mathrm{s} ; \quad v$ is X-ray frequency, unit is $\mathrm{Hz} ; c$ is the speed at which light travels in a vacuum, $c=3.0 \times 10^{8} \mathrm{~m} \cdot \mathrm{s}^{-1} ; \lambda$ is X-ray wavelength, unit is $\mathrm{m}$.

\section{$2.1 X$-ray attenuation characteristic}

When X-ray mutually effects with substance, different and difficult processes will appear. For its energy transformation, when X-ray passes substance, its energy will be separated to three parts: one will be scattered, another will be absorbed, and the last one will transmit in the original direction. After that, the X-ray intensity will be attenuated due to scattering and absorption.

Experiments indicated that the degree of X-ray attenuation varies directly with the distance of traveling substance, as shown in Figure 1. Assuming that intensity of incidence ray beam is $I_{0}$, after passing the substance with thickness $H$, the intensity is attenuated to $I_{H}$. A thin layer with thickness $d x$ is at depth $\mathrm{X}$ distance from incidence panel. When $\mathrm{X}$-ray pass $d x$, relative change of the intensity is

$$
\frac{I x-I_{x+d x}}{I x}=\frac{d I x}{I x}=-\mu d x
$$

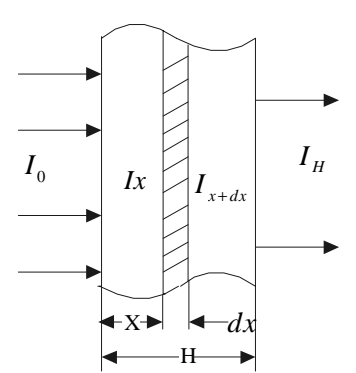

Figure 1. X-ray attenuation Principle

After passing the substance with thickness $\mathrm{H}$, the total attenuation of the light intensity is

$$
\int_{I_{0}}^{I_{H}} \frac{d I_{x}}{I_{x}}=-\mu \int_{0}^{H} d x \text {, that is } I_{H}=I_{0} e^{-(\mu / \rho) \rho H}=I_{0} e^{-\mu_{n} \rho H}
$$

where $\mu$ is line attenuation coefficient; $\rho$ is density of the substance; $\mu_{m}=\mu / \rho$ is mass attenuation coefficient that denotes the attenuation degree of unit weight substance against X-ray intensity, unit is $\mathrm{cm}^{2} / \mathrm{g}$. $\mu_{m}$ is irrelevant with substance status, but relevant with wavelength and atomic number $\mathrm{Z}$, that is $\mu_{m} \approx K \lambda^{3} Z^{3}$, where $\mathrm{K}$ is coefficient.

Provided that absorber is mixture or compound that consists with more than two elements, its total attenuation coefficient can be calculated by the expressions

$$
\mu_{m}=w_{1} \mu_{m 1}+w_{2} \mu_{m 2}+\cdots+w_{p} \mu_{m p}
$$

where $w_{1}, w_{2} \cdots w_{p}$ is weight percentage of each constituent; $\mu_{m 1}, \mu_{m 2} \cdots \mu_{m p}$ is mass attenuation coefficient of each constituent. The attenuation of X-ray intensity is processed by scattering and absorption, so mass attenuation coefficient $\mu_{m}$ should be equal to the sum of scattering coefficient $\sigma_{m}$ and absorption coefficient $\tau_{m}$, that is $\mu_{m}=\sigma_{m}+\tau_{m}$. In most cases, scattering coefficient is further smaller than absorption coefficient. $\mu=-1 n\left(I_{H} / I_{0}\right) / H$ expresses the intensity attenuation degree that X-ray spread in substance at unit length. For different $\mu_{m}, \mu$ and $I_{H}$ are different. Therefore, the X-ray nondestructive detection of different substance can be accomplished. This is the starting point that in our design sensing technique is adopted to implement nondestructive detection for conveyor belt. 
Distinct types of photo-electricity detectors have different concrete work principles, but they have electro-response to incidence light information and output photocurrent. (Gao, 1991). The ratio of output photocurrent and electron current of incidence light is quantum efficient. For consecutive X-ray spectrum, the quantum efficient is

$$
\eta=\frac{I_{p} / q}{P / h v}
$$

where $I_{p}$ is root mean square of electron current; $P$ is root-mean-square power of incidence light; $\mathrm{q}$ is electron quantity; $v$ is frequency of incidence light.

The factor which influences the spectrum response of photo-electricity diode is material absorption coefficient $\alpha(\lambda)$.Usually the absorption coefficient of shortwave $\alpha$ is great, so X-ray is absorbed at surface. However, composite action of surface against carries is highly strong. Photo-production carries can be compounded before cleaning out, so the quantum efficient is low. The cut-off wavelength $\lambda_{0}$ at light wave domain is decided by energy gap of semiconductor material, and $\lambda_{0}$ of $\mathrm{Si}$ photo-diode is $1.1 \mu \mathrm{m}$.

The wavelength of $X$-ray is $\lambda \approx 10^{-6} \mathrm{~cm} \sim 10^{-10} \mathrm{~cm}$. To photo-diode, the absorption coefficient $\alpha$ is great and the quantum efficient is low. For increasing the quantum efficient of Si photo-diode, radiation luminescence technique is adopted, and X-ray bombard luminescent substance to cause luminescence. In detector card used in this detector, structure matching of $C_{s} I(T I), G O S$ and $\mathrm{Si}$ photo-diode is adopted.

\section{X-ray nondestructive detection system for conveyor belt with steel wire ropes}

This detection system is composed of X-ray source, X-ray detector card, image pixel processing board, PC for running image detection system, power and so on. X-ray source emits cone-shaped beam of ray that change to fan-shaped after passing cross collimator; this fan-shaped beam of ray penetrates conveyor belt; and then it is received by one-dimensional X-ray detector card and becomes image pixel electrical signal, after collecting, conversing, transmitting and processing, the two-dimensional projection image of the conveyor belt can be achieved. These images can be dynamically displayed on screen of any PC at real-time that runs image detection program in network. If necessary, image can be saved, reappeared, zoomed out and system can alarm when super-standard. The system structure block diagram is shown in Figure 2.

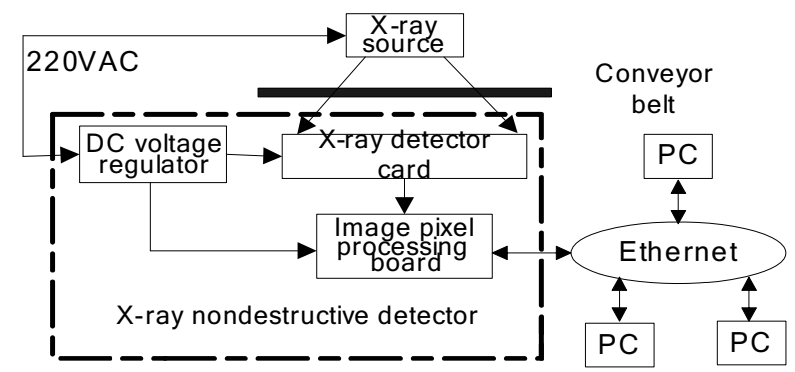

Figure 2. Nondestructive detection system structure block diagram

\section{Design of X-ray nondestructive detection system for conveyor belt with steel wire ropes}

This design consists of hardware and software. The hardware is namely design of nondestructive detector; and software separates into the software running on hardware platform and the software running on PC.

\subsection{Design of nondestructive detector}

Traditional nondestructive detection systems are mostly at single computer application moment, which can not connect to network. With the development of internet technique, SOPC technique and X-ray detection technique, network controlling and real-time remote image transmission of X-ray nondestructive detection system for conveyor belt with steel wire ropes become developing tendency. Therefore, we use VIRTEX-4 FPGA that built in PowerPC and MAC core of Xilinx Company and design a suit of high-speed, high-resolution and remote control X-ray nondestructive detection system for conveyor belt with steel wire ropes.

\subsubsection{X-ray detector card}

$\mathrm{X}$-ray detector card consists of Si photodiode arrays coated with a scintillator, integrating circuit and charge-sensitive amplifying circuit and so on. (Jussi, 2004). In application, the X-ray signals are absorbed by the scintillator array and converted to visible light. The visible light signals are detected by photodiode array. The electrical current from photodiode array is integrated in the read out integrating circuit by charge-sensitive amplifying circuit (one for each pixel). When triggered by ST, all pixel voltage signals can be read by serial module from X-ray detector card.

According to design requirement of nondestructive detection, X-CARD-32+32 is chosen to be the detection card. In this card, Pixel pitch of each Si photodiode is $1.5 \mathrm{~mm} \times 1.5 \mathrm{~mm}$. For inspecting all sections of conveyor belt once and the 
convenience of microcomputer image processing, this design uses 32 detection cards to place side by side in our design, which form about one dimensional array of $1.6 \mathrm{~m}$ and cover all sections of conveyor belt. The line scan frequency of this system is calculated by $f_{h}=v_{d} / 0.0015$, where $v_{d}$ is the speed of conveyor belt. For the detector card array, integration time is $0.2 \sim 10 \mathrm{~ms}$. It is suitable for the high speed design requirement of $4.3 \mathrm{~m} / \mathrm{s}$.

\subsubsection{Image pixel processing board}

Image pixel processing board consists of processor, ADC, and PHY and so on. In the design, A processor VIRTEX-4, working at frequency of $300 \mathrm{MHz}$, is selected for this system. According to the out-put voltage frequency of detection card and the high-speed, high-resolution and wide dynamic range of the detector, the ADS1610 with 10MSPS and 16-bit resolution operating from a $5 \mathrm{~V}$ analog and a $3 \mathrm{~V}$ digital supply is used as analog-to-digital converter (ADC). According to requirement of network data high-speed transmission, we choose Ethernet physical layer chip (88E1111) of Marvell Alaska. This chip is suitable for the usage of IEEE802.3 physical layer.

\subsubsection{Organization of PowerPC405}

The PowerPC405 is a 32-bit implementation of the PowerPC ${ }^{\circledR}$ embedded-environment architecture that is derived from the PowerPC architecture. Specifically, the PPC405 is an embedded PowerPC 405F6 (for VIRTEX-4) processor core. RISC processor of IBM PowerPC405 Harvard construction uses IP embedded technique to integrate to VIRTEX-E of Xilinx and gathers with other peripheral IP cores, which is realized system on chip (SOC). In Figure 3, its organization is shown.

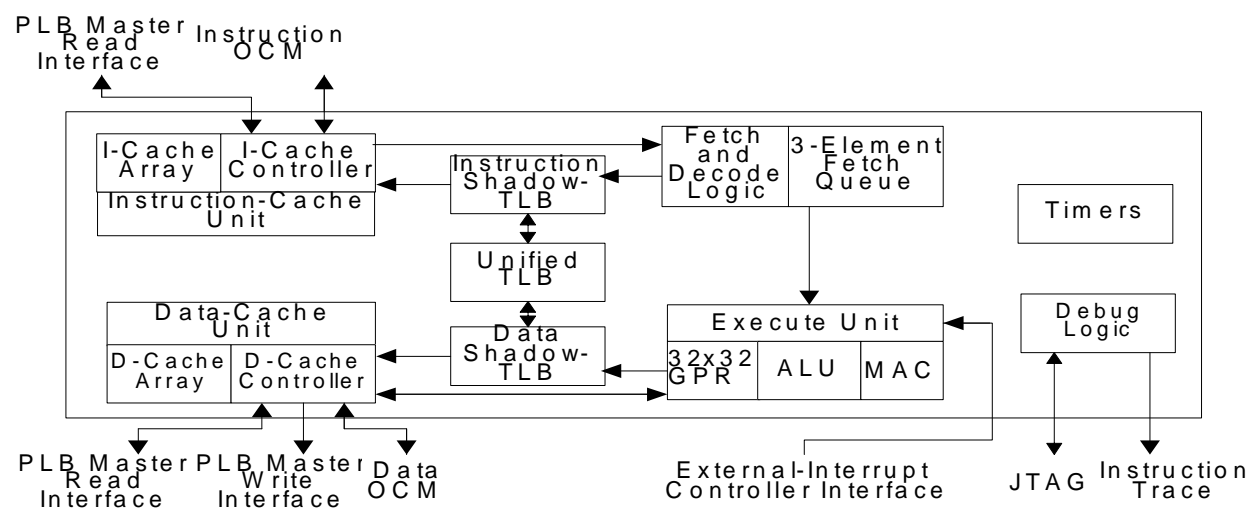

Figure 3. PPC405 organization diagram

The PowerPC 405 central-processing unit (CPU) implements a 5-stage instruction pipeline consisting of fetch, decode, execute, write-back, and load write-back stages. The PowerPC 405 processor has a thirty-two 32-bit registers that are accessed by the execute unit using three read ports and two write ports. The PowerPC 405 processor implements separate instruction-cache and data-cache arrays. Each is $16 \mathrm{~KB}$ in size, is two-way set-associative, and operates using 8 word (32 byte) cache lines. MMU is composed of variable pages sizes (1KB 16MB). The PowerPC 405 processor contains a 64-bit time base and three timers.

PowerPC405 processor is technically supposed by IBM Core-Connect that runs at 64 bits bus of 100 133MHz. For getting the maximally flexible system design, Core-Connect structure is implemented as soft IP core in VIRTEX-4 FPGA. Core-Connect bus structure has two main buses, which are processor local bus (PLB) and on-chip peripheral bus (OPB). These buses can be used to respectively connect high-speed and low-speed peripherals to PowerPC processor. Moreover, device control register bus managers and controls general peripheral equipment register.

PowerPC405 processor can response to the interrupt of software and hardware, and doing exception handling. (XILINX 2003). As well, the processor can debug processor system by using internal debugging module, external debugging module, debugging waiting module, real-time tracking module.

\subsubsection{Development process of PowerPC405}

The Xilinx Company provides Embedded Development Kit (EDK) for developing a complete embedded processor system based on the Xilinx ${ }^{\circledR}$ PowerPC ${ }^{\mathrm{TM}}$ and MicroBlaze ${ }^{\mathrm{TM}}$ which is implementation in a Xilinx FPGA device. (XILINX, 2003). Moreover, EDK provides the ability of software and hardware coordinated design, so that the design period can be greatly reduced. EDK supplies an integrated developed environment XPS (Xilinx platform studio) in order to use all tools supplied by system and accomplish the entire process of the embedded system development, which march ISE and FPGA objective board can implemented full-function development.

(1) Hardware development process 
Depending on Microprocessor Hardware Specification (MHS) and Microprocessor Peripheral Definition (MPD), embedded processor PowerPC405 and peripheral equipment IP core can be customized; external memorizer and other peripheral address space can be set; control signal of the peripheral equipment and cut-off signal can be managed. MHS can be generated by EDK tool and also can be written by user. EDK captures the hardware platform in the MHS file; MPD data files includes peripheral input-output port and parameter.

In this design, IP core provided by EDK tools, such as GPIO, SDRAM, UART, Ethernet MAC, is adopted to implement the connection of FPGA and other peripheral equipment. The structure of processor system in the nondestructive detector is shown in Figure 4.

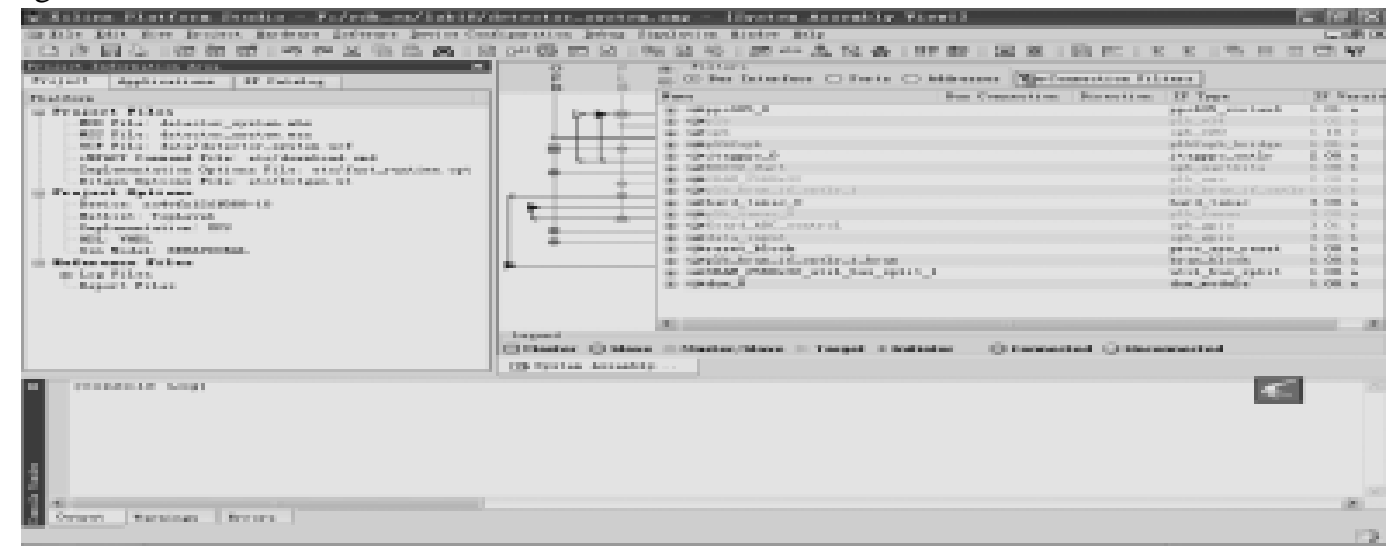

Figure 4. VIRTEX-4 FPGA system based on PowerPC405 structure block diagram

(2) Software development process

A software platform is a collection of software drivers and, optionally, the operating system on which to build application. The software image created consists only of the portions of the Xilinx library which is actually used in embedded design. EDK captures the software platform in the MSS file (Microprocessor Software Specification). XPS calls GNU compiler tools for compiling and linking application executables for each processor in the system. the compiler can read a set of $\mathrm{C}$ source and header files or assembler source files for the targeted processor. The linker combines the compiled applications with selected libraries and produces the executable file in ELF format. The Bitstream Initializer (Bitinit) tool initializes the on-chip BRAM memory connected to a processor with its software information. This utility reads hardware-only bitstream produced by the ISE tools (system.bit), and outputs a new bitstream (download.bit) which includes the embedded application executable (ELF) for each processor.

According to the function requires of the nondestructive detector, PowerPC405 is loaded LwIP, and application software is programmed in XilKernal system. (Que, 2006). This software mainly includes data acquisition program and network communicational program. Network communicational program adopted client and server model based on Socket to process network communication, and apply streaming formatter Socket to correspond to connection-objected TCP protocol in TCP/IP protocols. The network communicational program is shown in Figure 5. The nondestructive detector adopts XilKernal system to efficiently manage the teamwork between data acquisition program and network communicational program, and accomplish remote control and the real-time transmission of image data.

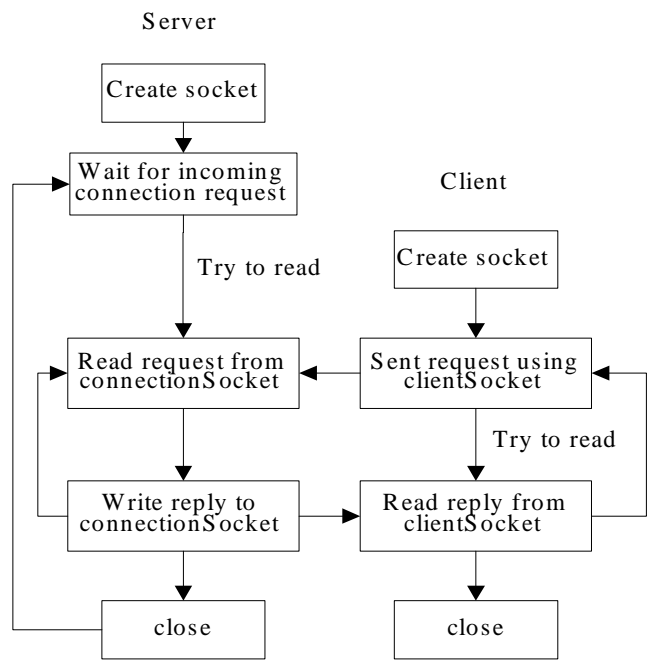

Figure 5. TCP network communication procedure 
4.1.3 Implementation of Nondestructive detector

$\mathrm{X}$-ray projection light signals are received by photodiode array of X-ray detector card, and a series of electric image signals are formed. After the electrical signals which are collected and converted by ADC are processed in VIRTEX-4 FPGA, a twodimensional projection image of the conveyer belt is obtained. The image data are transmitted to network. In Ethernet access part, image data are encapsulated according to IP protocol, and then data link layer protocol is used to accomplish the encapsulation of Ethernet frame to this protocol package. Eventually, image data are sent into Ethernet via net interface.

Nondestructive detector includes X-ray detector card, image pixel processor board and DC(digital current) voltage regulator. Image pixel processor board is the key part of the detector, which is composed of ADC, VIRTEX-4 FPGA, reset circuit, system clock circuit, memory circuit, PROM circuit, Ethernet PHY circuit and RS-232 interface, JTAG interface, LVPECL level interface circuit. Nondestructive detector structure is shown in Figure 6.

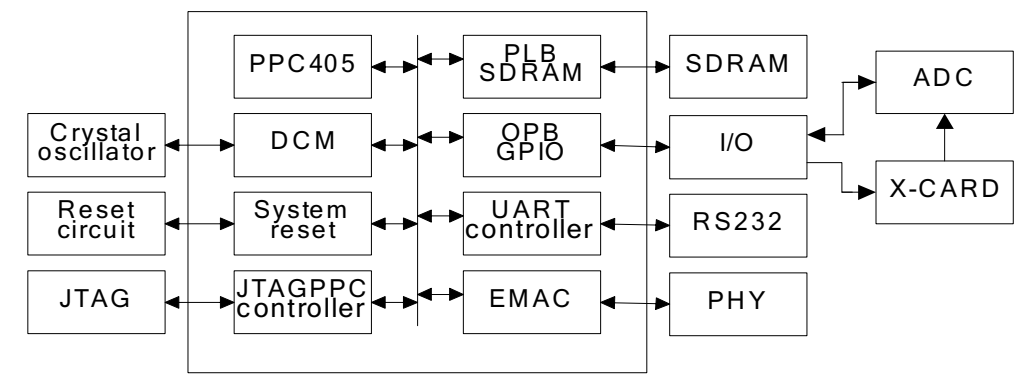

Figure 6. Nondestructive detector structure block diagram

\section{(1) System clock}

Reference clock is created by external crystal oscillator, and is connected with FPGA internal digital clock management module (DCM) and clock tree by global clock pin of FPGA. DCM supports more than 400MHz clock-out, and supplies a zero delay clock buffer that has 50/50 precision duty ratio. Meanwhile, DCM can accurately control 90, 180, 270 phase-shift ability, accomplishes synthesis of clock signal, and supports to create precise frequency of $24 \sim 420 \mathrm{MHz}$. In FPGA interior, DCM centrally distributes the clock signal. The clock output of DCM is set up to system clock of PowerPC, and supplied to PLB, OPB bus arbiter.

(2) External memorizer

PLB bus of PPC405 supports high-speed memory, such as DDR RAM, SDAM etc. OPB bus supports general storage circuit, such as EPROM, SRAM, FALSH and external register etc. In this design, 2 of high-speed synchronous SDRAM (MT48V8M16) are used to be external data memory area of PowerPC405 processor, which have 32 bits data bus width and $32 \mathrm{MB}$ capacity. When compiling MHS document, PLBSDRAM core, where memorizer address bus and data bus width are configured and initial address of memory area is appointed, is chosen to be add. Other setup of the program memorizer is similar as data memorizer besides linking to OPB bus.

(3) Ethernet interface

In FPGA, 10/100/1000M Ethernet MAC core is integrated and externally connected with Marvell Alaska Ethernet PHY(88E1111), which is suitable for the application of IEEE802.3 physical layer. And then it is connected to Ethernet after passing network isolation transformer. When MHS is compiled, Ethernet MAC core is chosen to be added.

(4) RS232 interface

As required, UART controller is integrated in FPGA interior, and is externally connected with RS232. Simultaneously, it links to computer serial that is used to be debugging information output of application program. In MHS, baud rate of serial, data bit width and parity check can be configured.

\section{(5) On-line programmable PROM}

FPGA, which is based on gate array, provides programmable recourse to users. Its logic structure is decided by configuration data. The configuration data are loaded to SRAM of FPGA interior by external control circuit or micro-processor. Due to the information stored in SRAM will lose when power-down, FPGA must be reconfigured when power-up. In practical application, 2 of PROM (XC18V04) are used to store configured data stream. FPGA is set to Master Serial mode via jumper. When power-up every time, the configuration data is automatically read from PROM to SRAM, and internal structure mapping is came true.

\section{(6) JTAG port}

The FPGA and PROM can be configured though JTAG port. When PowerPC is embedded in VIRTEX-4 FPGA, application code can be also downloaded and debugged. For the convenience of software code debugging, 4 of general I/O pins can also be appointed on FPGA besides special configured pins TCK, TMS, TDO and TDI in the FPGA. In 
addition, JTAG interface internally connected with PowerPC kernel is to configure and debug PowerPC kernel, and trace debug software code. In this module, software code can be singly downloaded to PowerPC kernel and debug software in kernel. When FPGA is configured via JTAG pins, the notice is that JTAG configuration pin would better connect with pullup resistor and INIT pin would better connect to earth as configuration period.

\subsection{PC software design}

General frame of developing image process software is set up in WINXP/WIN2000 platform by applying C\#.NET. Image process algorithm of conveyor belt is researched, and image processing software of conveyor belt is programmed.

4.2.1Image processing algorithm of conveying belt

Image processing algorithm of conveyor belt is used in the software development of image processing. (Roziere, 1989). Image processing algorithm of nondestructive detection system includes:

(1) Standardization method

Each pixel is adjusted in order to reduce noise adding of channel, and gain difference is eliminated that is caused by unevenness of crystal, coupling between crystal and photodiode or photodiode itself. Each pixel is adjusted by reapplying the equation:

$$
\text { Iiout=Gic (Iiraw-Oic+Ob/Gic) }
$$

Some variables and constants are listed as follows:

Iiout: standardization output signal of pixel i;

Gic: gain corrected value;

Iiraw: actual pixel value;

Oic: bias corrected value;

Ob: bias line value.

\section{(2) Grey enhancement}

Grey enhancement processing is to use grey transform technique to improve image quality by improving grey change of image. In some cases, grey distribution range of image just centralized in narrower area that causes worse contrast and vague outline of image. At that time, the image grey distribution can be extended to 256 grey degree (0 255) by linear transform in order to improve entire contrast and resolution of image. Assumed that grey distribution arrange of original image is A1-A2, the equation of transformation is $\mathrm{g}(\mathrm{x}, \mathrm{y})=255 /(\mathrm{A} 2-\mathrm{A} 1)[\mathrm{f}(\mathrm{x}, \mathrm{y})-\mathrm{A} 1]$. In addition, due to proper nonlinear grey transform (such as logarithm, exponent), a target of grey arrange in image can be excel.

\section{(3) Edge sharpening}

Sharpening is to emphasize image contour and make image clear and legible. Essence of sharpening is high-pass filtering technique. Image sharpening can be implemented by template operation (operator). Choosing property template can accomplish edge sharpening, boundary detection, feature extraction and smoothed filter and so on.

(4) Image inversion

According to the detection theory of X-ray, X-ray passing steel wire are less than surrounding colloid matter, so image signal is relatively weak and image is relatively dark. Image hue of X-ray is inversed to make shown image fit observation habit of detection personnel. Assumed that grey values of pixel point before and after inversion are respectively $f(x, y), g$ $(\mathrm{x}, \mathrm{y})$, the inversion equation is: $\mathrm{g}(\mathrm{x}, \mathrm{y})=255-\mathrm{f}(\mathrm{x}, \mathrm{y})$.

In development of software, a lot of methods will be used, such as nonlinear median filtering, edge detection, discrete Fourier transform and inverse transform, fast Fourier transform (FFT) and inverse transform etc.

\subsubsection{Image processing software of conveyor belt}

The development of conveyor belt image processing software consists of dynamic display module, defect detection module, data management module, and image processing module and so on.

Dynamic display module mainly completes initialization of detector and show-card, and accomplishes detection images dynamic display, capture, reconstruction, Zoom-in and roaming and so on.

Defect detection module completes fuzzy recognition algorithm based on image space character (space contrast and space variance), which is to be defect detection algorithm. Initially, advanced image processing method is adopted to make place and defect more extrusive and striking, and then depending on automatic identification system of computer, quantitative evaluation, hierarchical classification and qualified judgment system of defect is constructed. Artificial Intelligence algorithm is introduced.

Data management module manages detected image data, and reasonably sets database and stores according to different joint control numbers or failure numbers and different collective times. Simultaneously, inquire, browsing and modification of database is supplied. 
Image processing module uses digital image processing technique to enhance contrast and definition of image, aiming at definition enhancement algorithm, such as internal geometric rectification, grey transform, pseudo color. Diagnostic area prefabricates plot functions toolbox, measurement toolbox and text editor. By using it, users can increase necessary assistant information on the images; simultaneously, users are allowed to store data and share dynamic data stream through internet.

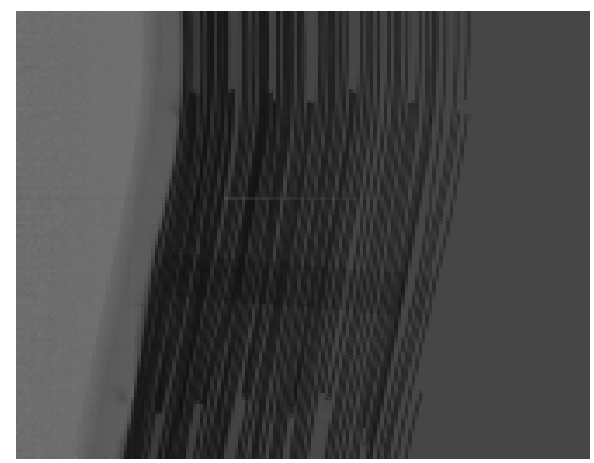

Figure 7. The image collected by nondestructive detector

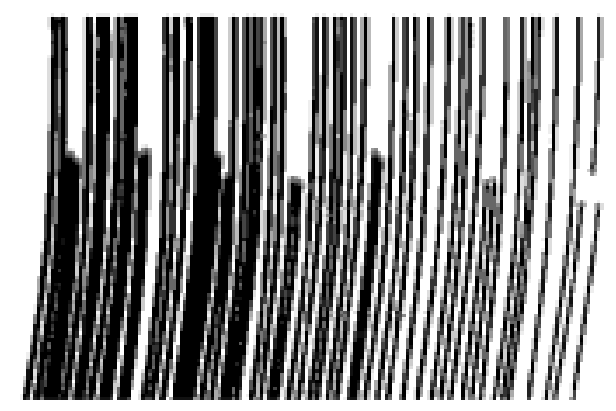

Figure 8 . The image processed by image processing software

\section{Conclusion}

In this paper, X-ray detection theory is used to design nondestructive detection system for high-speed running conveyor belt with steel wire ropes. At the case that the speed of conveyor belt is $4.3 \mathrm{~m} / \mathrm{s}$, nondestructive detection system implements high-speed detection and static running, which is eligible for design requirement. This detection system accomplishes remote control and network transmission of image data, and improves automatically degree of nondestructive detection system. Furthermore, staff can be far from radiant source environment, and it is easy to achieve radiant protection and personnel safety.

\section{References}

Cheng, Hong, Gao, yulin \& Zhao, Shujing. (1997). A X-ray Non-destructive Test System for Mine Conveyer Belt. High Technology Letters. Vol.3, No.1, June, 26 29.

Gao, Zhonglin \& Wang, Kaiyuan. (1991). Photoelectric Devices. Nanking: Publishing House of Southeast university. Getting Started with EDK. (2003). XILINX.

Jussi Koskinen. (2004). LDA Technology Today Possibilities in the Future. Proc. CT \& 3D Imaging Conference. $132 \sim 137$.

Klug, H.P. \& Alexander, L.E.. (1974). X-ray Diffraction Procedures for Polycrystalline and Amorphous Materials. PowerPC Processor Reference Guide. (2003). XILINX.

Que, Dashun \& Wang, Jintao. (2006). Implementation of LwIP in the $\mu$ c/OS-II. Ship Electronic Engineering. Vol.26, No.4, 89 91.

Roziere B M et al. (1989). High Resolution Solid State Linear Detector for X-ray Digital Imaging, Non-Destructive Testing, Proc. $12^{\text {th }}$ World Conference. 705. 